\title{
Enhancing collaborative practice-based research in an academic health science center
}

\author{
Lianne Jeffs ${ }^{* 1,2}$, Beverly Bulmer ${ }^{2}$, Maria Maione ${ }^{2}$, Sharon Straus ${ }^{1,2}$, Ella Ferris ${ }^{2}$ \\ ${ }^{1}$ Keenan Research Centre, Li Ka Shing Knowledge Institute, St. Michael's Hospital, Toronto, Canada \\ ${ }^{2}$ St. Michael's Hospital, Toronto, Canada
}

Received: September 14, 2017

Accepted: November 19, $2017 \quad$ Online Published: November 29, 2017

DOI: $10.5430 /$ jnep.v8n4p51

URL: https://doi.org/10.5430/jnep.v8n4p51

\begin{abstract}
Although there is a body of literature around discipline specific capacity building research, less is known empirically around collaborative practice-based research that involves different healthcare disciplines participating in research together in an interprofessional manner in Academic Health Science Centers (AHSCs). This paper provides an overview of results from the mixed methods study and the subsequent strategies one teaching hospital has implemented to enhance collaborative practicebased research. A mixed methods design with a cross-sectional survey design and focus groups/interviews with clinicians and administrators was used. Study participants reported the value of, their role, experience, and use of research in daily practice and recommendations to enhance collaborative practice-based research. Study findings elucidated the current state and strategies to enhance collaborative practice-based research at an AHSC. Engaging clinicians in collaborative practice-based research provides a strategic advantage and return on investment to achieve the tripartite mission.
\end{abstract}

Key Words: Interprofessional research, Mixed methods, Capacity building

\section{INTRODUCTION}

Academic Health Science Centers (AHSCs) have the tripartite mission of excellence in research, practice, and education. ${ }^{[1]}$ Key to achieving this mission is integrating research, practice, and education and aligning with the operational activities in AHSCs. ${ }^{[1]}$ Further, a focus on collaborative applied research including disciplinary specific and interprofessional research is critical to achieving quality outcomes and cost efficiencies in health care and a global priority. ${ }^{[2-5]}$ Interprofessional research focuses on generating clinically relevant knowledge of collaborative teams from different healthcare professional backgrounds ${ }^{[6]}$ working with patients, their families, caregivers and communities (referred to collaborative practice-based research). Healthcare professionals working within AHSCs are well positioned to carry out collaborative practice-based research due to their proximity to patients and opportunity to generate clinically driven research questions $;{ }^{[5,7]}$ particularly nurses and health disciplines that collectively comprise the largest healthcare workforce within health care. ${ }^{[5]}$

There are reported benefits around engaging nurses and health disciplines in research in healthcare organizations including increased research productivity, efficiency and reduced patient mortality and morbidity; ${ }^{[8,9]}$ uptake of evidence $;^{[9,10]}$ increased job satisfaction ${ }^{[11]}$ and reduced staff turnover. ${ }^{[8,9]}$ However, although there is a body of literature around discipline specific capacity building research, $\left.{ }^{4}, 10,12\right]$ there is less known empirically around collaborative practice-

\footnotetext{
*Correspondence: Lianne Jeffs; Email: jeffsl@smh.ca; Address: Li Ka Shing Knowledge Institute, St. Michael’s Hospital, Toronto, Ontario, M5B 1W8, Canada.
}

Published by Sciedu Press 
based research; particularly in Canada. Research involvement from nurses; ${ }^{[13,14]}$ and health disciplines ${ }^{[4,7,15]}$ continues to remain low. Key to engaging nurses and health disciplines in collaborative practice-based research is having an understanding of the current context that they can be used to develop a strategic approach to building research capacity. ${ }^{[7,12]}$ In this context, a mixed methods study was undertaken to establish a baseline and gain insight into nurses' and health disciplines' engagement in research as part of developing a strategic approach to enhancing collaborative practice-based research at one academic hospital.

\section{MethodS}

The study employed a two-phased design that consisted of an initial cross-sectional survey with mainly closed ended questions with a few open-ended questions. To gain further insight into nurses' and health disciplines' perceptions and experiences associated with being involved in research in the hospital, the second phase consisted of presenting the survey results to study participants in either an interview or focus group guided by a series of open-ended questions. The study was conducted in a 463-bed inner city tertiary hospital in the city of Toronto with a total of 6,066 staff of which approximately 1,900 are regulated health disciplines. Developing practice-based research capacity was identified as a strategic direction in the interprofessional practice strategic plan at a large teaching hospital that two of the co-authors (BB, LJ) had corporate oversight for advancing. The hospital's Research Ethics Board (REB) approved the study.

\subsection{Survey}

An email with the link to the online survey was sent to all healthcare professionals and administrators in May 2014. Reminder emails were sent four times throughout the month of May to encourage staff to answer the survey. Inclusion criteria for participants included that they were regulated healthcare professionals (nurses and health disciplines) employed at the hospital and able to provide informed consent. Alcock, Carroll and Goodman' ${ }^{[16]}$ Staff Nurses and Research Activities instrument was adapted and replaced the word nurse with healthcare professionals (except physicians) with no changes made to the intent of the original survey questions. The authors pilot tested the instrument and reported high internal consistency for the five scales (value of nursing research, expectations, interest in nursing research, research experience and organizational climate) with alpha coefficients of 0.81 , $0.71,0.87,0.79$ and 0.78 , respectively. Also, questions on infrastructure, capacity building, and preferences for research and knowledge translation were included. The survey asked participants a series of questions in each of the domains and used both close-ended using a 7 point Likert scale or yes- no binary response and open-ended with an opportunity for participants to provide other comments. An example question was "Research-based knowledge assists me in solving patient care problems; it is the healthcare professional's role to be involved in research if it addresses ways to improve the quality of patient care; administration (directors, middle managers) is supportive of health care professionals who conduct research." Completion of the survey served as implied consent. No identifying information was provided by survey participants and results were aggregated. Descriptive, analytical methods were employed to analyze the survey data. Open-ended comments were analyzed using a content analysis approach where the most frequently cited comments are aggregated into categories.

\subsection{Focus groups and interviews}

A purposive sample was recruited from the administration (Clinical Leader Managers, Clinical and Professional Practice Directors) and through the nursing and health disciplines councils (Nursing Advisory Council and Health Disciplines Advisory Council) to participate in focus groups and interviews during April-September 2014. Informed consent was obtained from study participants by a research coordinator before conducting the focus groups or interviews. A slide deck of the survey results and a semi-structured interview guide was used. Key questions included: What do you think of these findings? Do they resonate with you? Why or Why not? Focus groups and interviews were transcribed and analyzed using a content analysis methods. ${ }^{[17,18]}$

A total of 29 healthcare professionals participated in the focus groups and interviews. As some health care professionals could not join the scheduled focus groups, individual interviews were scheduled at a mutually convenient time. Of the 29 healthcare professionals that participated in the focus groups and interviews 55\% were health disciplines and $45 \%$ were Registered Nurses (RNs). The health disciplines were represented by physiotherapists, occupational therapists, speech language pathologists, medical lab technologists, respiratory therapists. $38 \%(\mathrm{n}=5)$ of the RNs were Nurse Practitioners. Interviews were transcribed with all identifying information removed by the principal investigator prior to analysis. Transcripts are being stored separately from the completed informed consent forms.

\section{RESUltS}

\subsection{Demographic profile}

\subsubsection{Survey}

Of the 242 survey respondents (representing a $12.7 \%$ response rate $), 85.1 \%(\mathrm{n}=206)$ were female, the majority, $81.8 \%(\mathrm{n}=198)$, were employed on a full-time basis, while 
$12.8 \%(\mathrm{n}=31)$ were part-time employees and $5.4 \%(\mathrm{n}=13)$ were employed on a casual basis. Survey respondents came from a wide variety of professional backgrounds with registered nurses comprising the largest segment of the sample at $49.2 \%(\mathrm{n}=119)$. The remainder of professional backgrounds included in the survey can be seen in Table 1 .

Table 1. Professional background

\begin{tabular}{ll}
\hline Professional Background & Frequency (\%) \\
\hline Registered Nurse & $49.2 \%,(\mathrm{n}=119)$ \\
Medical Laboratory Technician/Technologist & $7.9 \%,(\mathrm{n}=19)$ \\
Physical Therapist & $6.2 \%,(\mathrm{n}=15)$ \\
Pharmacist & $5.4 \%,(\mathrm{n}=13)$ \\
Registered Dietitian & $5.0 \%,(\mathrm{n}=12)$ \\
Respiratory Therapist & $4.5 \%,(\mathrm{n}=11)$ \\
Nurse Practitioner & $3.7 \%,(\mathrm{n}=9)$ \\
Clinical Nurse Educator & $2.9 \%,(\mathrm{n}=7)$ \\
Social Worker & $2.5 \%,(\mathrm{n}=6)$ \\
Speech Language Pathologist & $2.5 \%,(\mathrm{n}=6)$ \\
Spiritual Care Provider & $2.5 \%,(\mathrm{n}=6)$ \\
Occupational Therapist & $1.7 \%,(\mathrm{n}=4)$ \\
Clinical Nurse Specialist & $1.7 \%,(\mathrm{n}=4)$ \\
Vascular Ultrasound Technologist & $0.8 \%,(\mathrm{n}=2)$ \\
Medical Radiation Technologist & $0.8 \%,(\mathrm{n}=2)$ \\
Cardiovascular Technologist (ECG) & $0.4 \%,(\mathrm{n}=1)$ \\
Audiologist & $0.4 \%,(\mathrm{n}=1)$ \\
Advanced Practice Physiotherapist & $0.4 \%,(\mathrm{n}=1)$ \\
MRI Technician & $0.4 \%,(\mathrm{n}=1)$ \\
Ultrasound Technologist & $0.4 \%,(\mathrm{n}=1)$ \\
Echocardiography Technician & $0.4 \%,(\mathrm{n}=1)$ \\
Physical Therapy Assistant & $0.4 \%,(\mathrm{n}=1)$ \\
TOTAL & $100.0 \%,(\mathrm{n}=242)$ \\
\hline & \\
&
\end{tabular}

\subsubsection{Focus groups/interviews}

Four focus groups and four interviews were conducted that included the following participants $(\mathrm{N}=19)$ : two program directors; two managers; four nurse practitioners; two registered nurses; and a social worker, laboratory technologist, physiotherapist, pharmacist, occupational therapist, respiratory therapist, speech-language pathologist, dietician, and radiation technologist.

Results are presented under the following survey domains-value of, role and interest in; experience with research; research culture and environment; and preferences for infrastructure and learning format. Clinicians and administrators reported how valuable collaborative practicebased research is at the hospital to improving clinical practice $(88.8 \%)$, solving patient care problems $(84.7 \%)$, guiding clinical decisions (84.3\%), and promoting accountability for practice $(83.5 \%)$. Survey respondents also described having interest in knowing the results of research projects that have been conducted in the workplace $(94.2 \%)$, changing clinical practice on research findings (91.3\%), finding answers to specific clinical practice problems $(89.7 \%)$, participating in research projects if they are carried out in one's workplace $(86.8 \%)$, reading about research studies $(83.9 \%)$, and conducting research if it is part of the work assignment (82.6\%). The priority areas for collaborative practice-based research focused on patient outcomes and experience, care transitions, mobility and team collaboration. As noted by a program director "Everything should be centred around the patient, so it is not surprising. The distribution is probably reflective of the engagement and interest in research."

Survey respondents and focus group/interview participants identified several roles associated with collaborative practicebased research that mainly focused on improving the quality of patient care and clinical practice as outlined in Table 2 . The need to have clarity around the roles and expectations of clinicians in research emerged in the focus groups/interviews. As one nurse described "They're [healthcare workers] not sure what their roles are in terms of research, and they sometimes feel that it's more at the bedside as opposed to research, I think that people don't know their roles in terms of how they can be involved in research." One health discipline suggested to "include research as an expectation in job descriptions."

Table 2. Role in research

\begin{tabular}{lll}
\hline Role in Research & Average & Frequency (\%) \\
\hline Suggest ways to improve patient care & 1.69 & $95.5 \%$ \\
Find ways to solve patient care problems & 1.87 & $95 \%$ \\
Identify patient care problems & 1.74 & $93 \%$ \\
Apply research findings to clinical practice & 2.05 & $90.1 \%$ \\
Be involved in research if it addresses ways to improve the quality of patient care & 2.06 & $89.3 \%$ \\
Be involved in collecting data for research studies & 2.68 & $75.6 \%$ \\
Be aware of all research being conducted in the workplace & 2.66 & $75.2 \%$ \\
Conduct research studies & 3.12 & $62.8 \%$ \\
Involved in collecting data only if it can be incorporated into the daily practice & 3.19 & $60.3 \%$ \\
\hline
\end{tabular}


The most common experience with research reported was serving as study participants $(88.8 \%)$. Respondents reported less experience leading research teams and conducting research including collecting and analyzing data. Table 3 provides a list of all the survey responses to experiences with research. These findings were supported by the focus groups and interviews. However, some participants thought that "presenting research findings at conferences" should have been a higher percent due to all the internal opportunities to present research.

Table 3. Experience with research

\begin{tabular}{|c|c|}
\hline Experience with Research & Frequency (\%) \\
\hline Serving as study participants & $88.8 \%(n=213)$ \\
\hline Taking courses in statistics & $78.5 \%(n=189)$ \\
\hline Changing clinical practice based on research findings & $78.5 \%(n=189)$ \\
\hline Taking courses in research methodology & $69.8 \%(n=169)$ \\
\hline Developing a questionnaire & $63.2 \%(n=153)$ \\
\hline Attending research conferences & $46.3 \%(n=112)$ \\
\hline Analyzing quantitative data & $45.0 \%(\mathrm{n}=109)$ \\
\hline Analyzing qualitative data & $44.2 \%(n=107)$ \\
\hline Identifying a clinical problem which led to a research study & $41.3 \%(n=100)$ \\
\hline Collecting specimens for a research project & $40.9 \%(n=99)$ \\
\hline Serving as a co-investigator of a research project & $34.7 \%(n=84)$ \\
\hline Conducting interviews for a research project & $33.9 \%(n=82)$ \\
\hline Presenting research findings at conferences & $27.7 \%(n=67)$ \\
\hline Conducting a focus group & $24.4 \%(n=59)$ \\
\hline Assisting in writing a grant proposal & $24.0 \%(n=58)$ \\
\hline Developing an interview guide & $21.5 \%(\mathrm{n}=52)$ \\
\hline Serving as principal investigator & $16.5 \%(n=40)$ \\
\hline Publishing research results & $16.5 \%(n=40)$ \\
\hline Leading the writing of a grant proposal & $8.3 \%(n=20)$ \\
\hline Receiving funds as a principal investigator to conduct research & $7.4 \%(n=18)$ \\
\hline
\end{tabular}

Survey respondents identified the research culture and environment was supportive of clinicians being engaged in collaborative practice-based research at a moderate to less than moderate range (72.7\%-33.1\% respectively). The strongest support for collaborative practice-based research was reported coming from physicians $(72.7 \%)$, clinicians $(66.1 \%)$, senior management $(58.3 \%)$, followed by administration (directors and middle managers) (55.0\%). Variation in support was also identified in the focus groups and interviews as noted in the following two narratives:

"Physicians and administration is supportive because [our hospital] is geared toward research. We have the [research institute] which fosters research development." (Nurse)

"On some units, nurses are always called on to do research with backfill and health disciplines are never asked." (Health Discipline)

"We have to be more mindful of how to build research activities into their day to day." (Manager)
The most commonly reported preferred infrastructure resource for participating in collaborative practice-based research was having protected time and designated funds for clinicians to perform research activities within their scheduled work. The need for formal, dedicated research positions including research coordinators and clinician scientists were also identified. A few respondents noted that there should be equitable distribution amongst nursing and health disciplines. Other resources identified included having access to mentors with expertise in research methods to guide clinicians to develop research study designs and proposals, statisticians to assist with data analysis, assistance to navigate the institutional REB protocol submissions, and encouragement and support from directors, clinical leader-managers, and professional practice leads for clinicians to engage in collaborative practice-based research.

Content areas to focus on for capacity building for collaborative practice-based research included how to 1) analyze data 
$(76.4 \%) ; 2)$ conduct research $(73.6 \%) ; 3)$ publish research $(71.1 \%) ; 4)$ present research $(70.2 \%) ; 5)$ write a research proposal (69.8\%); and 5) write a REB proposal (62.8\%). Participants identified specific strategies for building collaborative practice-based research and knowledge translation capacity that included providing funds for pilot studies and small research, formal workshops and training, and dissemination of research findings particularly research that has been conducted at the hospital. Table 4 provides preferences for infrastructure for collaborative practice-based research identified by survey respondents.

Table 4. Infrastructure/learning format preferences

\begin{tabular}{ll}
\hline Content Area & Frequency (\% yes) \\
\hline Series of workshops on research & $77.7 \%$ \\
Presentation of completed research & $75.2 \%$ \\
Series of workshops on knowledge translation strategies & $74.0 \%$ \\
On-line modules & $71.1 \%$ \\
Working-in-progress-research & $66.9 \%$ \\
Formal knowledge translation program & $60.7 \%$ \\
Formal research capacity program & $55.8 \%$ \\
\hline
\end{tabular}

\section{Discussion}

Our study aimed at examining clinicians' (nurses and health disciplines) and administrators' perspectives collaborative practice-based research at one hospital offers empirical evidence on the current state of collaborative practice-based research. Specifically, in our study, clinicians' experience with research was mainly a study participant, taking researchrelated courses, and research utilization compared to conducting research. This finding points to the lack of knowledge, competence, and barriers to engage in researching daily clinical practice consistent with the literature. ${ }^{[2,4,10,13,15,19,20]}$ Our study elucidated how clinicians valued the use of research to solve patient care problems, answer clinical questions, and guide clinical decisions and to improve patient care, in their daily practice. Previous studies involving clinicians also found that their interests with research are primarily to produce knowledge that is clinically relevant and is used to enhance safety and quality care to achieve the best outcomes for their patients. ${ }^{[7,12,14,21]}$

The need for a multi-pronged approach to build collaborative research capacity (e.g., a variety of strategies and access to expertise) emerged from our study. Our study participants felt strongly that protected time for research was required to enhance clinicians' engagement in research consistent with other empirical work on the need for protected time for clinicians to develop research competence and engage in research efforts integrated into their scheduled work. ${ }^{[12,14,22]}$ Our study participants also described the need for having secondment opportunities where they are mentored by a research expert. Other authors have identified research supervision, mentorship, and partnerships as prerequisites for enhancing clinicians' research skills and competencies. ${ }^{[2,12,21]}$ Other supports required to enhance the engagement in collaborative practice-based research included having formal, dedicated research positions (e.g., research coordinators, clinician scientists) similar to other literature. ${ }^{[2,5,12,15,23]}$ Further, one systematic review identified the need to integrate point-ofcare research into organizations in the form of continuous, stable activities and policies that ensure the sustainability and further development of clinical research projects. ${ }^{[14]}$

Interestingly, clinicians viewed that their physician colleagues were most supportive with the management being viewed less supportive. This finding may be due in part to physicians who work in academic teaching hospitals value research with many having scientist roles and affiliations with the hospital and university who conduct research. The perceived less support from management to engage in research warrants further attention as leaders are essential in establishing a research culture and evidence-based practice setting. ${ }^{[14]}$ Golenko et al. (2012) ${ }^{[2]}$ identified influencers to building research capacity as being an organization-wide approach and support from senior managers as well as organizational structures, processes, and systems. Some study participants identified inequalities between disciplines in research opportunities (e.g., nurses having more opportunity to engage in research) that also requires further attention. A partial explanation is that the hospital that the study was conducted in had earmarked funds to build research capacity with nurses from the provincial government as part of the Nursing Enhancement Funds and through a series of grants obtained by the Director of Nursing Research. ${ }^{[12]}$ There is also evidence to suggest that funding for health discipline research is inequitable as compared to other disciplines (e.g., 
nurses and physicians) internationally. ${ }^{[2,4,7]}$

The low response rate, exclusion of physicians, and the conduct of the study at one hospital are limitations that may render the study results not generalizable to other hospitals.

\section{Moving forward: Our action plan}

Using a research capacity building framework, the results of the mixed methods study, and relevant literature (see examples of references in brackets under elements of the framework), the following collaborative practice-based research action plan was developed. Key underpinning principles included 1) the need to ensure that authentic collaborative practice-based research would be enacted that included collaborative teams from different professional backgrounds generating clinically relevant knowledge working with patients, their families, caregivers and communities ${ }^{[5]}$ and 2) equitable opportunities for all clinicians (nurses and health disciplines) to participate in collaborative practice-based research. The research capacity building framework was developed in the rehabilitation health sector ${ }^{[24]}$ and used to build nursing research capacity at the same hospital. ${ }^{[12]}$ Within this framework there are five core inter-related elements:

(1) Develop a cadre of researchers. ${ }^{[12,25]}$

(2) Create a vibrant research culture, environment, and infrastructure. ${ }^{[5,12,23]}$

(3) Secure funding. ${ }^{[2,24]}$

(4) Establish and strengthen partnerships with scientists in other disciplines and academic departments. ${ }^{[21,26]}$

(5) Identify and evaluate metrics. ${ }^{[12,24]}$

Specific recommended actions are outlined in Table 5.

Table 5. Collaborative practice-based research (CPBR) plan of action

\begin{tabular}{|c|c|}
\hline Capa & Reco \\
\hline $\begin{array}{l}\text { Develop a Cadre of } \\
\text { Researchers }\end{array}$ & $\begin{array}{l}\text { - Understand the current state of Ph.D.; Masters prepared researchers within hospital who can facilitate/partner with } \\
\text { clinicians } \\
\text { - Understand and support discipline-specific growth interest in CPBR aligned with corporate priorities } \\
\text { - Explore internships/fellowships; clinician scientist and research associate roles aligned with CPBR }\end{array}$ \\
\hline $\begin{array}{l}\text { Create a Research } \\
\text { Culture and } \\
\text { Infrastructure }\end{array}$ & $\begin{array}{l}\text { - Establish a governance structure with aligned working groups to oversee the CPBR and knowledge translation } \\
\text { action plan and selection of CPBR projects } \\
\text { - Develop a communication plan to increase awareness of the vision for and resources/strategies for enhancing } \\
\text { CPBR } \\
\text { - Provide monthly CPBR rounds for sharing of internal and external CPBR } \\
\text { - Create sustainable CPBR workshop series with situated and on-line learning aligned with interprofessional and } \\
\text { discipline-specific CPBR projects. All projects have demonstrated alignment with } \\
\text { corporate/programmatic/discipline specific priorities } \\
\text { - Develop and evaluate learning and return on investment from a CPBR capacity grant competition } \\
\text { - Create a manager of health disciplines CPBR position } \\
\text { - Develop an online strategy for CPBR and knowledge translation including adapting/converting the Nursing } \\
\text { Research Advancing Practice toolkit to on-line modules and other linkages }\end{array}$ \\
\hline $\begin{array}{l}\text { Establish/ } \\
\text { Strengthen } \\
\text { Partnerships }\end{array}$ & $\begin{array}{l}\text { - Increase collaboration with internal partners-Applied Health Research Centre, Knowledge Translation Program, } \\
\text { Research Ethics Board, Quality Improvement Department, Health Sciences Library, etc. to establish clear } \\
\text { partnerships/linkages to enable ongoing support/mentorship for CPBR and knowledge translation } \\
\text { - Increase collaboration and opportunities to leverage research interests and strengths of academic faculty } \\
\text { - Consider clinician scientist positions that are a result of partnerships between academic affiliates and programs } \\
\text { - Leverage partnerships and capitalize on research projects (post-doctoral); thesis projects (Ph.D. or Masters), } \\
\text { practicums at graduate level, and undergraduate research programs }\end{array}$ \\
\hline Secure Funding & $\begin{array}{l}\text { - Collaborate with the hospital and foundation to secure funding for small pilot/seed grants for CPBR competition } \\
\text { - Develop a plan to secure grant opportunities from different external sources applicable to CPBR projects }\end{array}$ \\
\hline Identify and & - Finalize analysis of baseline including publications, presentations, and Research Ethics Board submissions \\
\hline Evaluate & - Set targets annually for CPBR and knowledge translation \\
\hline Metrics & - Conduct an annual review of capacity building, publications, presentations and Research Ethics Board submissions \\
\hline
\end{tabular}

A strategic, phased approach ${ }^{[12,27]}$ focusing on strengthening existing processes and leveraging partnerships is being employed. ${ }^{[7,10,27]}$ Efforts are underway in all five of the research capacity domains including a series of training and workshop opportunities (alternating monthly research profiling and capacity building lunch sessions) and a small grant competition 
aligned with current corporate safety and quality strategic priorities (September 2015 - September 2017). Efforts are also underway to develop a measurement framework to determine the return on investment of collaborative practice-based research.

\section{Conclusion}

Our study provided empirical evidence around the current state of collaborative practice-based research at an AHSC. Key results included that clinicians saw value in conducting and using research to solve problems, answer questions, and guide clinical decisions to evaluate the impact on patient care. However, inequities amongst health disciplines and nurses were reported. Study findings informed the development of a strategic approach to enhancing collaborative practice-based research at one academic acute care hospital. Having clinicians engage in collaborative practice-based research (in addition to discipline-specific research) provides a strategic advantage and return on investment to organizations as a workplace culture that emphasizes collaboration and teamwork, innovation, and research capabilities with increased job satisfaction and productivity of the workforce-ultimately resulting in reduced morbidity and mortality and positive patient outcomes. Continued investment by healthcare leaders is required to develop collaborative practice-based research competence and capacity to generate and apply knowledge that enhances quality patient care and collaborative practice and achieves the tripartite mission of AHSCs.

\section{ACKNOWLEDGEMENTS}

We would like to acknowledge the nurses, health disciplines and hospital administrators that participated in the study. We would also like to thank Orla Smith and Nabeel Hack for their contribution to the initial literature reviews and Joyce Lo and Christopher Stewart for their contribution to data collection and analysis.

\section{Conflicts of InTERest Disclosure}

The authors declare that there is no conflict of interest.

\section{REFERENCES}

[1] King G, Thomson N, Rothstein M, et al. Integrating research, clinical care, and education in academic health science centers: An organizational model of collaborative workplace learning. Journal of Health Organization and Management. 2016; 30(7): 1140-1160. PMid:27700478 https : //doi .org/10 . 1108/JHOM-11-2015-0 177

[2] Golenko X, Pager S, Holden L. A thematic analysis of the role of the organisation in building allied health research capacity: a senior managers' perspective. BMC Health Services Research. 2012; 12(276): 110. Available from: http://www.biomedcentral .com/1472-6 963/12/276 https://doi.org/10.1186/1472-6963-12-276

[3] Hulcombe J, Sturgess J, Souvlis T, et al. An approach to building research capacity for health practitioners in a public health environment: an organisational perspective. Australian Health Review. 2014; 38(3): 252-8. PMid:24785693 https : //doi .org/10.1071/AH13066

[4] Pickstone C, Nancarrow S, Cooke J, et al. Building research capacity in the allied health professions. Evidence \& Policy: A Journal of Research, Debate and Practice. 2008; 4(1): 53-68. https : //doi.org/10.1332/174426408783477864

[5] Wenke R, Mickan S. The role and impact of research positions within healthcare settings in allied health: A systematic review. BMC Health Services Research. 2016; 16(355). https ://doi.org/10.1186/ s12913-016-1606-0

[6] Martin JS, Ummenhofer W, Manser T, et al. Interprofessional collaboration among nurses and physicians: making a difference in patient outcome. Swiss Medical Weekly. 2010; 140: w13062. https://doi.org/10.4414/smw.2010.13062

[7] Pager S, Holden L, Golenko X. Motivators, enablers, and barriers to building allied health research capacity. Journal of Multidisciplinary Healthcare. 2012; 5: 53-59. https ://doi.org/10.2147/JMDH.S 27638

Published by Sciedu Press
[8] Harding K, Lynch L, Porter J, et al. Organisational benefits of a strong research culture in a health service: a systematic review. Australian Health Review. 2016. [Epub ahead of print].

[9] Zallman L, Tendulkar S, Bhuyia N, et al. Provider's perspectives on building research and quality improvement capacity in primary care: A strategy to improve workforce satisfaction. Clinical and Translational Science. 2013; 6: 404-408. https ://doi.org/10.1111/ct s. 12066

[10] Cooke J. A framework to evaluate research capacity building in health care. BMC Family Practice. 2005; 6(1): 44. https ://doi .org/10 $.1186 / 1471-2296-6-44$

[11] Black AT, Balneaves LG, Garossino C, et al. Promoting evidencebased practice through a research training program for point-of-care clinicians. Journal of Nursing Administration. 2015; 45(1): 14-20. https://doi.org/10.1097/NNA.0000000000000151

[12] Jeffs L, Smith O, Beswick S, et al. Investing in nursing research in practice settings: A blueprint for building capacity. Canadian Journal of Nursing Leadership. 2013; 26(4): 44-59. https ://doi .org/10 $.12927 /$ cjnl.2013.23630

[13] Kelly KP, Turner A, Speroni KG, et al. National survey of hospital nursing research, Part 2: facilitators and hindrances. Journal of Nursing Administration. 2013; 43(1): 18-23. https : //doi.org/10.1 $097 /$ NNA . Ob013e3182786029

[14] Lode K, Sørensen EE, Salmela S, et al. Clinical nurses' research capacity building in practice-A systematic review. Open Journal of Nursing. 2015; 5(07): 664-667. https ://doi .org/10.4236/ojn. 2015.57070

[15] Wenke RJ, Ward EC, Hickman I, et al. Allied health research positions: a qualitative evaluation of their impact. Health Research Policy and Systems. 2017; 15(6). https://doi.org/10.1186/s12961 $-016-0166-4$ 
[16] Alcock D, Carroll G, Goodman M. Staff nurses' perceptions of factors influencing their role in research. The Canadian Journal of Nursing Research. 1990; 22(4): 7-18. PMid:2134646

[17] Ryan GW, Bernard HR. Data management and analysis methods. In N. Denzin and Y. Lincoln, (2nd ed.). The SAGE Handbook of Qualitative Research. Thousand Oakes, California: Sage Publications; 2000 .

[18] Ryan GW, Bernard HR. Techniques to identify themes. Field methods. 2003; 15(1): 85-109. https://doi.org/10.1177/152582 2X02239569

[19] Stephens D, Taylor N, Leggat SG. Research experience and research interests of allied health professionals. Journal of Allied Health. 2009; 38(4): 107E-111E.

[20] Syme R, Stiles C. Promoting nursing research and innovation by staff nurses. Applied Nursing Research. 2012; 25(1): 17-24. https: //doi.org/10.1016/j.apnr.2010.06.005

[21] Akerjordet K, Lode K, Severinsson E. Clinical Nurses Attitudes Towards Research, Management and Organizational Readiness in a University Hospital: Part1. Journal of Nursing Management. 2012; 20: 814-823. PMid:22967299 https ://doi.org/10.1111/j.13 $65-2834.2012 .01477 . \mathrm{x}$
[22] Harding KE, Stephens D, Taylor NF, et al. Development and evaluation of an allied health research training scheme. Journal of Allied Health. 2010; 39(4): e143-8. PMid:21184016

[23] Wilson B, Kelly L, Reifsnider E, et al. Creative approaches to increasing hospital-based nursing research. Journal of Nursing Administration. 2013; 43(2): 80-88. PMid:23343724 https ://doi.org/ 10.1097/NNA. Ob013e31827f 2043

[24] Frontera WR, Fuhrer MJ, Jette AM, et al. Rehabilitation medicine summit: building research capacity executive summary. Assistive Technology. 2006; 18(1): 2-14. https ://doi.org/10.1080/10 400435.2006.10131902

[25] Duffy JR, Culp S, Yarberry C, et al. Nurses' research capacity and use of evidence in acute care: Baseline findings from a partnership study. Journal of Nursing Administration. 2015; 45(3): 158-164. https://doi.org/10.1097/NNA.0000000000000176

[26] Whitworth A, Haining S, Stringer H. Enhancing research capacity across healthcare and higher education sectors: development and evaluation of an integrated model. BMC Health Services Research. 2012; 12(287). https : //doi .org/10.1186/1472-6963-12-287

[27] Trostle J. Research capacity building in international health: definitions, evaluations, and strategies for success. Social Science Medicine. 1992; 35: 1321-24. https://doi.org/10.1016/0277-9536(92 ) $90035-0$ 